\title{
BMJ Open Immigrant screening for latent tuberculosis infection: numbers needed to test and treat, a Norwegian population-based cohort study
}

\author{
Brita Askeland Winje, ${ }^{1}$ Gry Marysol Grøneng, ${ }^{2}$ Richard Aubrey White, ${ }^{2}$ Peter Akre, ${ }^{3}$ \\ Preben Aavitsland, ${ }^{4,5}$ Einar Heldal ${ }^{6}$
}

To cite: Winje BA, Grøneng GM, White RA, et al. Immigrant screening for latent tuberculosis infection: numbers needed to test and treat, a Norwegian population-based cohort study. BMJ Open 2019;9:e023412. doi:10.1136/ bmjopen-2018-023412

- Prepublication history and additional material for this paper are available online. To view please visit the journal (http:// dx.doi.org/10.1136/bmjopen2018-023412).

Received 7 April 2018 Revised 5 November 2018 Accepted 7 November 2018

Check for updates

(C) Author(s) (or their employer(s)) 2019. Re-use permitted under CC BY-NC. No commercial re-use. See rights and permissions. Published by BMJ.

For numbered affiliations see end of article.

Correspondence to Dr Brita Askeland Winje; brita.winje@fhi.no

\section{ABSTRACT}

Objectives To estimate the number needed to screen (NNS) and the number needed to treat (NNT) to prevent one tuberculosis (TB) case in the Norwegian immigrant latent tuberculosis infection (LTBI) screening programme and to explore the effect of delay of LTBI treatment initiation.

Design Population-based, prospective cohort study. Participants Immigrants to Norway.

Outcome Incident TB.

Methods We obtained aggregated data on immigration to Norway in 2008-2011 and used data from the Norwegian Surveillance System for Infectious Diseases to assess the number of TB cases arising in this cohort within 5 years after arrival. We calculated the average NNS and NNT for immigrants from the top 10 source countries for TB in Norway and by estimated TB incidence rates in source countries. We explored the sensitivity of these estimates with regard to test performance, treatment efficacy and treatment adherence using an extreme value approach, and assessed the effects of emigration, time to TB diagnosis (to define incident TB) and intervention timing. Results NNS and NNT were overall high, with substantial variation. NNT showed numerically stronger negative correlation with TB notification rate in Norway $(-0.75$ [95\% Cl -1.00 to -0.44$]$ ) than with the WHO incidence rate $(\mathrm{IR})(-0.32$ [95\% $\mathrm{Cl}-0.93$ to 0.29$])$. NNT was affected substantially by emigration and the definition of incident TB. Estimates were lowest for Somali (NNS 99 [70-150], NNT 27 [19-41]) and highest for Thai immigrants (NNS 585 [413-887], NNT 111 [79-116]). Implementing LTBI treatment in immigrants sooner after arrival may improve the effectiveness of the programme.

Conclusion Using TB notifications in Norway, rather than IR in source countries, would improve targeting of immigrants for LTBI management. However, the overall high NNT is a concern and challenges the scale-up of preventive LTBI treatment for significant public health impact. Better data are urgently needed to monitor and evaluate NNS and NNT in countries implementing LTBI screening.

\section{BACKGROUND}

The WHO has issued guidelines for the programmatic management of latent
Strengths and limitations of this study

- A population-based and sensitive surveillance system provided national data on all new cases of tuberculosis (TB).

- Country-specific administrative data were used to estimate person-time under observation for immigrants.

- We applied different estimates of latent TB test sensitivity, treatment efficacy and adherence to treatment to calculate uncertainty.

- The prevalence of latent TB infection in recent immigrants was estimated from published surveys rather than individual data.

- Some cases of TB present on arrival may have been misclassified as having onset after arrival.

tuberculosis infection (LTBI). ${ }^{12}$ The guidelines strongly recommend screening for and treatment of LTBI in groups at high-risk for tuberculosis (TB) and conditionally in recent immigrants from high to low TB incidence countries. ${ }^{1}$ LTBI is common and the risk of progression to TB varies substantially among individuals, assumed to reflect age, time since infection and host immune status. ${ }^{1}$

The identification of target immigrant groups for LTBI management remains challenging in most low TB incidence settings. There has been a call for the harmonisation of migrant screening policies across Europe. ${ }^{3}$ Eligibility for screening is commonly based on the TB incidence rate (IR) in the country of origin or the reason for immigration, with typical focus on asylum seekers and refugees. ${ }^{3}$ It has, however, been suggested that the targeting of immigrants based on the TB IR in the host country may improve the effectiveness of immigrant screening programmes. ${ }^{4}$

In Norway, foreign-born individuals account for almost $90 \%$ of TB notifications, and the majority are diagnosed in the first 5 
years after arrival. ${ }^{5}$ Based on molecular surveillance of Mycobacterium tuberculosis strains, the majority of TB in the foreign-born population is assumed to reflect reactivation of LTBI acquired prior to arrival. ${ }^{5}$ Against this backdrop, Norway has a well-established immigrant screening programme for TB and LTBI. Immigrants are currently targeted for TB screening based on the WHO-estimated TB IRs in their countries of birth. ${ }^{6}$ Immigrants younger than 35 years are also targeted for LTBI management to prevent future development of TB. The eligibility for arrival LTBI screening has differed over time; in March 2017 the IR cut-off value was changed from $>40 / 100000$ to $>200 / 100000$ (including immigrants from Afghanistan and Eritrea). ${ }^{7}$ The monitoring and evaluation system of the long-standing TB and LTBI screening programme is weak.

The primary objective of this study was to use aggregated numbers of Norwegian immigration and individual-level TB surveillance data to estimate the number needed to screen (NNS) and the number needed to treat (NNT) with LTBI chemoprophylaxis to prevent one TB case in the immigrant LTBI screening programme. The secondary objectives were to estimate the number of TB cases prevented by the current strategy in a 4-year cohort of immigrants and to explore the effect of delay of LTBI treatment initiation within the first 6 months versus the 12 months after arrival, using the same immigration and surveillance data.

\section{METHODS}

\section{Data sources and creation of data set for modelling and} analysis

We combined aggregate numbers from Norwegian immigration data (ie, information on the entire cohort) and individual-level TB surveillance data (ie, information on individuals with TB or LTBI treatment) to create a unified data set for modelling and analysis. All steps are described in the text below. A complete overview is also presented in table format in online supplementary appendix $1 \mathrm{a}-\mathrm{d}$.

\section{Data and sources}

Immigration and emigration data

We have used administrative data on immigration by year, country of origin and reason for immigration in Norway in 2008-2011. Data were obtained separately from two different sources: the Norwegian Directorate of Immigration (UDI) for newly arrived asylum seekers and from Statistics Norway (SSB) for other immigrant groups. The number of immigrants is based on the number of asylum applications and the number of residence permits for other immigrant groups. The country of origin reflects citizenship for asylum seekers and country of birth for other immigrant groups. We estimated the proportion aged $<15$ years and $15-35$ years by country, reason for immigration and year of immigration based on the reported age distribution from SSB/UDI (online supplementary appendix 1a). As emigration from Norway is substantial in some immigrant groups, we obtained aggregated administrative data on time spent in Norway before emigration from the same sources (further described below). In the model, we have assumed that immigrants who received residence permit or applied for asylum actually immigrated to Norway and that immigrants who were later registered as emigrated, or had a final rejection of application for asylum, actually emigrated (online supplementary appendix 1c).

\section{TB cases and LTBI treatment}

For individuals with TB and LTBI treatment (ie, the people of interest), individual-level demographic and clinical information was obtained from the Norwegian Surveillance System for Infectious Diseases (MSIS) for the years 2008-2016. This time period allows for 5-year observation time for all immigrants. Information included age at notification, country of birth, date of notification, date of diagnosis (collection of clinical sample) and date of start of treatment. Further, on the MSIS notification form, clinicians report time in Norway prior to diagnosis for foreign-born individuals using the following categories: <1 month, 1-6 months, 7-12 months, 1-2 years, 3-4 years, $5-9$ years and $>10$ years. The date of arrival is not reported.

It is mandatory for laboratories and clinicians to report TB diagnosis and treatment outcome, and prescription of LTBI treatment, to MSIS. Untreated LTBI is not reported. The sensitivity of MSIS data is assumed to be high because notifications are sent from multiple sources and are checked routinely against $\mathrm{TB}$ drug prescriptions.

We used all TB notifications to MSIS in 2008-2015 (year of reporting) to identify the top 10 source countries (in absolute numbers; online supplementary appendix 2) for immigrant TB in Norway and then calculated the TB notification rate (NR) in Norway based on the number of observation years.

\section{Construction of analysis data set}

Based on the aggregated immigration data, we calculated the number of arriving immigrants aged $\leq 35$ years from the top 10 source countries for TB in Norway and for countries with WHO-estimated TB IRs >150/100 000 population in the period 2008-2011. We used the WHO Global TB Report 2014 estimates of TB IR in countries of origin in $2013 .{ }^{6}$

\section{Estimated prevalence of LTBI}

We used a positive Interferon Gamma Release Assay (IGRA) as a proxy for LTBI. The prevalence of IGRA positives was based on published literature, including Norwegian data on asylum seekers, ${ }^{8}$ and ranged from $18 \%$ to $29 \%$, depending on the WHO-estimated TB IR in the country of origin and the age group 0-14 years and 15-35 years. ${ }^{8-10}$ The number of immigrants with LTBI in the model was estimated by multiplying the number of arriving immigrants with the published estimates of IGRA positives, separately for the two age groups. In the model 
we have assumed that the age-specific and country-specific prevalence of LTBI from published literature, including Norwegian data, is a fair proxy for the LTBI prevalence in the arrival cohort.

\section{TB and LTBI treatments in the 2008-2011 immigrant cohort}

We used the categorical information about time in Norway prior to diagnosis from MSIS to estimate a probability distribution for each case's arrival year in Norway (eg, 'a case received a diagnosis in December 2010 and has been in Norway for $<1$ month, therefore they have $100 \%$ probability that they arrived in Norway in 2010 and belong to the 2008-2011 immigrant cohort', 'a case received a diagnosis in March 2012 and has been in Norway for 1-6 months, therefore they have a $50 \%$ probability that they arrived in Norway in 2011, and $50 \%$ probability that they arrived in Norway in 2012'). When information about the time since arrival was missing, we imputed this information by applying the country-specific probability distribution for time in Norway. We then estimated the number of individuals with TB or LTBI treatment who belonged to the 2008-2011 cohort of immigrants by multiplying the number of cases by the probability that they immigrated to Norway in 2008-2011.

We excluded individuals who were diagnosed with TB (based on the date of sample collection for TB diagnosis) within 1 month after arrival, as these individuals were most likely ill on arrival (coprevalent TB) and TB would not be preventable through LTBI screening and treatment. For sensitivity analysis, we also excluded individuals who were notified within 1-6 months. These cases may or may not have been preventable through LTBI management. Based on this uncertainty, we present NNS and NNT separately for TB diagnosed $>1$ and $>6$ months after arrival, and applied these two definitions of incident TB throughout the study.

\section{Estimation of time in Norway}

Since emigration is substantial in some immigrant groups, we estimated the cumulative probability of time under observation in Norway based on the UDI/SSB administrative data. For asylum seekers, data on emigration were obtained as percentile distributions of the number of days from application date to the date of the final rejection of application; for example, among 421 asylum seekers from Somalia who arrived in Norway in 2008 and whose application for asylum later was rejected, $10 \%$ were rejected within 62 days, $20 \%$ were rejected within 87 days and so on up until the $90 \%$ percentile. We used this information to calculate the number of person-years of observation lost due to emigration within the first 5 years after arrival in Norway. This was done separately by country, TB IR in the country of citizenship and by year.

For other immigrant groups, data on emigration were based on aggregated September 2014 data, containing the number of immigrants per year and the number of them that emigrated before September 2014 (separately by reason for immigration) (see table 1 for an example of the data and the formulae used to estimate the cumulative probability distribution for duration of time in Norway for the cohort).

\section{Finalising data set}

Using the prior pieces of information (number of people arriving each year, probability distribution of time to emigration, and for each TB/LTBI diagnosis, time since immigration and estimated year of arrival), we created a data set containing yearly cohorts of people who immigrated to Norway between 2008 and 2011 and are followed up for either 5 years or until they emigrate from Norway (the shorter of the two).

\section{Outcomes}

\section{Preventable TB/risk of preventable TB}

We defined preventable TB as a patient notified with TB to MSIS and who (1) arrived to Norway in 2008-2011, (2) was notified to MSIS $>1$ month (6 months) and $<5$ years after arrival, and (3) was younger than 40 years of age at notification (to allow for 5-year observation time after screening). With this relatively short time period, we assume that they were infected prior to arrival in Norway. We explored the sensitivity of these estimates regarding test performance, treatment efficacy and adherence to treatment using an extreme value approach. IGRA sensitivity was estimated to be $84 \%$ (with $81 \%$ and $87 \%$ applied as extreme values), ${ }^{11} 12$ and chemoprophylaxis efficacy was estimated to be $65 \%(50 \%-80 \%),{ }^{13}$ consistent with a UK study. ${ }^{4}$ The rate of treatment adherence was estimated to be $90 \%(80 \%-100 \%)$, based on previous studies, including Norwegian data. ${ }^{14-17}$ The number of incident TB cases was adjusted accordingly and defined as preventable TB (table 2). We excluded TB cases that were on TB treatment on arrival to Norway.

Table 1 The cumulative probability distribution for duration of time in Norway for immigrants other than asylum seekers

\begin{tabular}{|c|c|c|c|c|}
\hline Year of arrival $(\mathrm{X})$ & $\begin{array}{l}\text { Number arrived in } \\
\text { year } X\end{array}$ & $\begin{array}{l}\text { Number } \\
\text { emigrated before } \\
\text { September } 2014\end{array}$ & $\begin{array}{l}\text { Average time } \\
\text { in Norway as of } \\
\text { September } 2014\end{array}$ & $\begin{array}{l}\text { Cumulative proportion } \\
\text { staying in Norway as } \\
\text { of September } 2014\end{array}$ \\
\hline 2008 & D1 & N1 & 6.25 & 1-N1/D1 \\
\hline 2009 & D2 & N2 & 5.25 & 1-N2/D2 \\
\hline 2010 & D3 & N3 & 4.25 & $1-\mathrm{N} 3 / \mathrm{D} 3$ \\
\hline 2011 & D4 & N4 & 3.25 & $1-N 4 / D 4$ \\
\hline
\end{tabular}




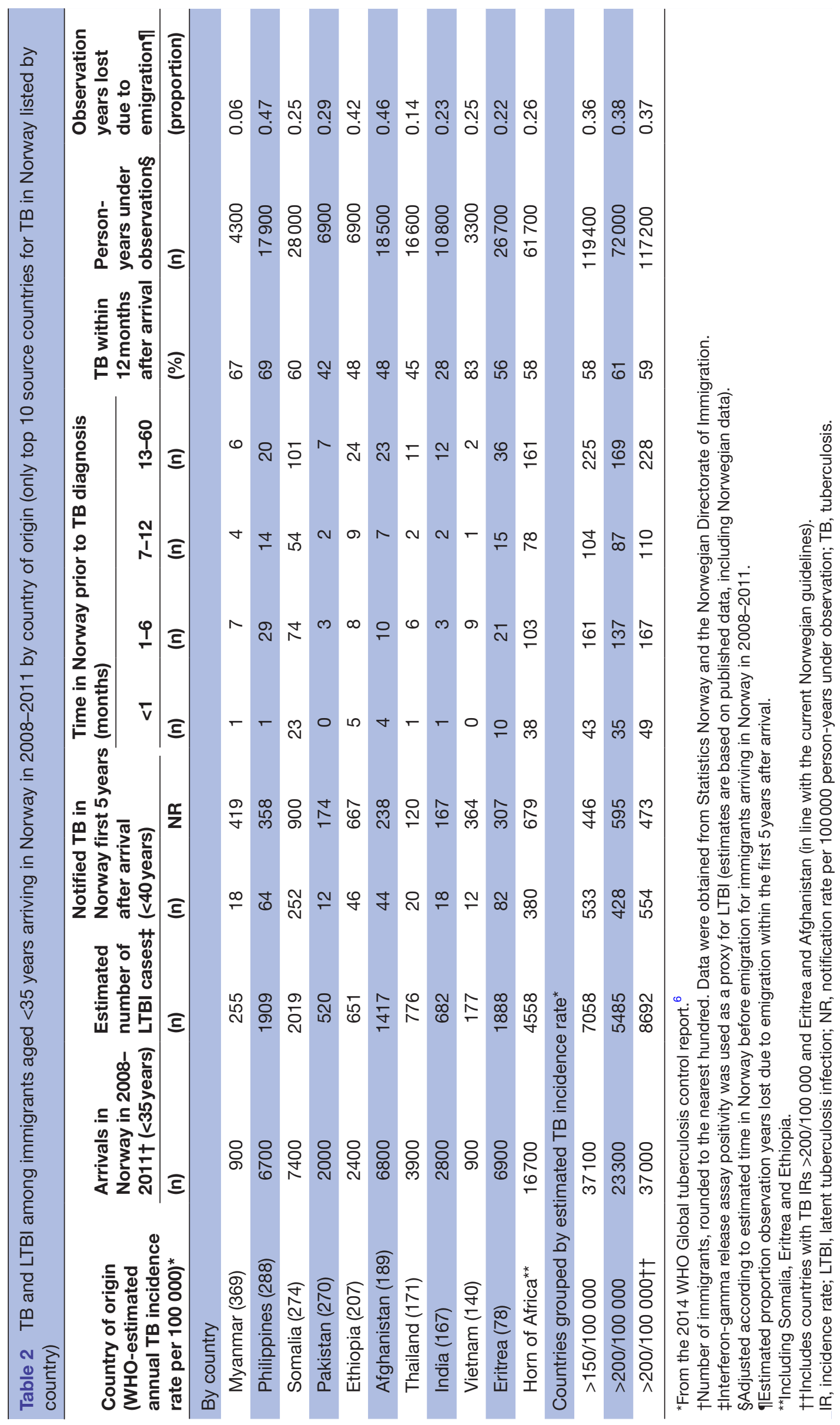


For each time period after arrival to Norway $(<1$ month, 1-6 months, 7-12 months, 1-2 years, 3-4 years, 5-9 years and $>10$ years), we obtained the number of preventable TB cases and then calculated the risk of preventable TB per time period (ie, number of cases divided by number of people). The risk of developing TB during this time period was then also converted into monthly risk using the formula $1-(1 \text {-total risk })^{\wedge}(1 /$ number-months $)$.

\section{NNS and NNT}

We estimated the NNS to prevent one incident TB case by calculating the ratio of the number of arriving immigrants to the number of preventable TB cases observed in Norway within 5 years. We used the extreme value approach to explore the sensitivity of these estimates.

We estimated the crude NNT as the ratio of the number of individuals testing positive for LTBI to the number of preventable TB cases. This NNT can be interpreted as a combined effect of emigration and TB risk (ie, if someone emigrates from Norway, they cannot receive a TB diagnosis in Norway, thus the more emigration the lower the risk for TB observed in Norway). We used the information on person-years lost for observation due to emigration to calculate corrected NNT as $1 /$ (risk of preventable TB in 5 years). This number can be interpreted as the NNT if all immigrants remained in Norway for 5 years.

We then explored correlation with 95\% CIs of the NNT with the TB NR in Norway and WHO-estimated TB IR. The purpose of this analysis was to identify which data source (TB NR in Norway or WHO-estimated TB IR) had a stronger association with public health implications in Norway (NNT).

Prevented TB due to LTBI treatment and the effect of delay of LTBI treatment initiation

We estimated the expected number of TB prevented by the LTBI treatments provided during the study period. This was calculated by multiplying the number of LTBI treatments by the subsequent risk of preventable TB in different time periods (based on the categorical MSIS data on time since arrival). The calculations were limited to the first 5 years in Norway (eg, if a person received LTBI treatment after 4 years in Norway, LTBI treatment would have a preventive effect for only 1 year). In the model, we have assumed that all immigrants eligible for screening actually were screened and that they were screened soon after arrival in line with the mandatory screening programme. We further assumed that a person did not leave Norway after receiving LTBI treatment. Calculations were based on incident TB $>1$ month after arrival.

We calculated the percentage increase in prevented TB (potential for additional prevention) when LTBI treatment was initiated within the first (1) 6 months and (2) 12 months after arrival to Norway (based on the $84 \%$ sensitivity $/ 65 \%$ treatment effectiveness $/ 90 \%$ adherence estimates and incident TB $>1$ month after arrival) by multiplying increased number of people screened by sensitivity by effectiveness by adherence. The outcome reflects a combination of the timing of TB diagnosis and LTBI treatment, or a strong effect of one of them.

\section{Uncertainty in the calculations}

None of the calculations in this study included uncertainty. Our model was primarily deterministic. The source of uncertainty in our study came from running our deterministic model with alternative IGRA sensitivities and treatment efficacies (the extreme value approach).

\section{Patient and public involvement}

Patients and or the public were not involved in the study.

\section{RESULTS}

The majority of foreign-born patients with TB in Norway originated from the Horn of Africa; Somalia alone accounted for $44 \%$ of TB cases from the top 10 source countries (table 2). Overall, a high proportion of TB occurred within the first year after arrival, with some variation among source countries. The fraction of observation years lost due to emigration was substantial in some groups and varied among source countries (table 2).

Most immigrants from the Horn of Africa, Afghanistan and Myanmar arrived as refugees and asylum seekers (figure 1). Most immigrants from Vietnam, Thailand and Pakistan arrived for family reunification, whereas immigrants from India arrived for family reunification and work, and the majority of immigrants from the Philippines came to work as au pairs.

Overall, estimated NNS and NNT were high (table 3). Estimates were lowest for Somalia: screening of 70-150 and treatment of 19-41 Somali immigrants were required to prevent one incident TB case (6-month threshold for preventable TB). NNT was lowest for estimates corrected for the effect of emigration and with the 1-month threshold to define incident TB, compared with the crude NNT and the 6-month threshold (table 3). The same pattern was seen for all countries. NNT was highest for immigrants from Pakistan and Thailand, although NNS was substantially higher for Thailand. For most source countries, the number of preventable TB cases was reduced by one-third when the 6-month definition of incident TB was applied compared with the 1-month definition, but with variation (range $16 \%-75 \%$ ).

We found a stronger numerical correlation between the TB NR in Norway and NNT to prevent one incident TB case (correlation coefficient [CC] -0.75 [95\% CI -1.00 to $-0.44]$ ) than between the NNT and WHO-estimated IR in the country of origin (CC -0.32 [95\% CI -0.93 to 0.29$]$ ) for the top 10 source countries for TB in Norway (using corrected NNT and the 6-month definition of incident TB). The CCs were affected only modestly by emigration and definition of incident TB, and unaffected by the extreme value approach (data not shown). The WHO-estimated TB IRs in Somalia and Pakistan in 2013 were similar (274 and 270/100 000 person-years). These values contrast with our findings that NNT was lowest for Somali 


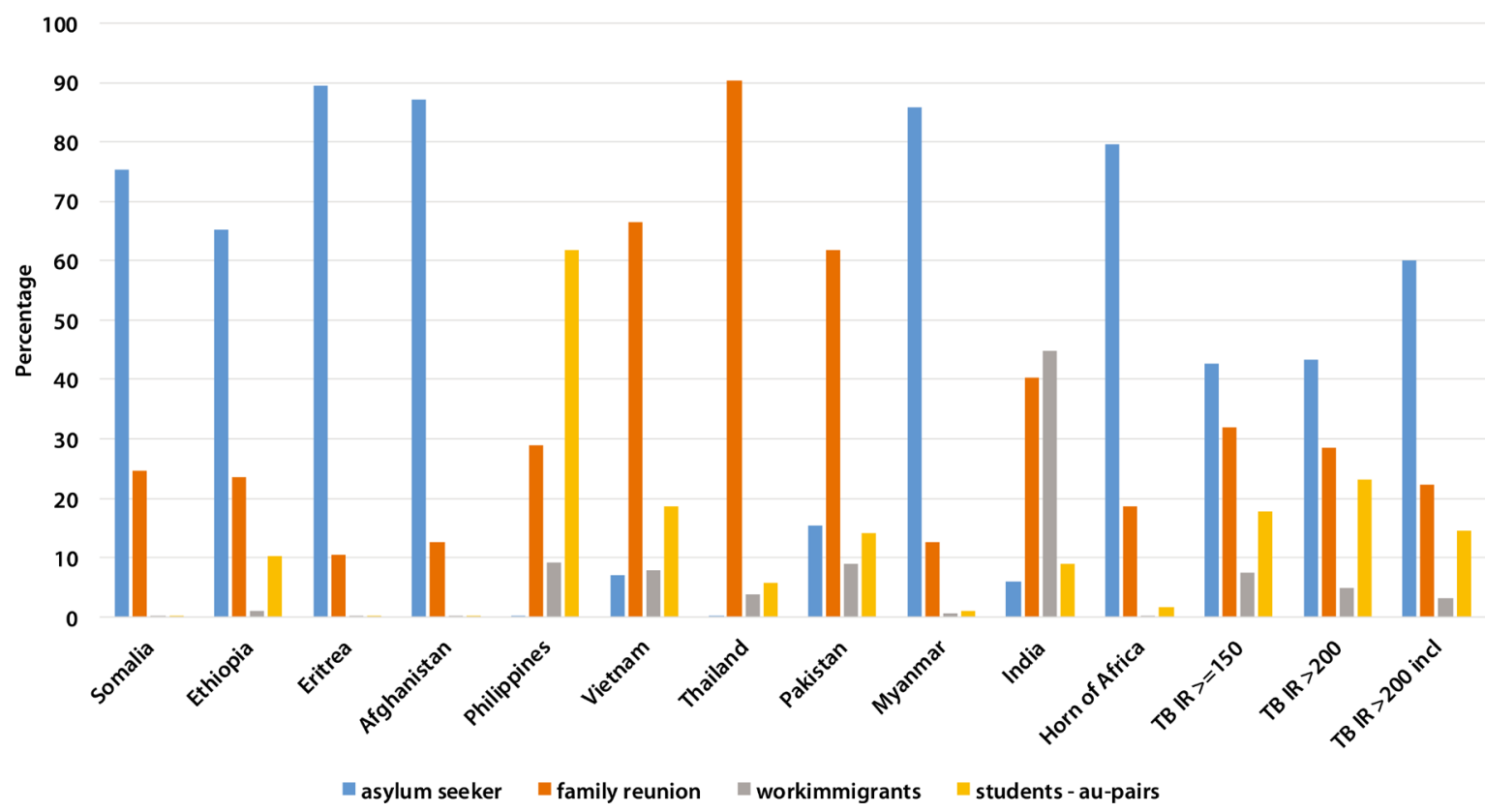

Figure 1 Reasons for immigration for immigrants aged $<35$ years arriving in Norway in 2008-2011, by country of origin (\%). IR, incidence rate; TB, tuberculosis.

immigrants and the highest for Pakistani immigrants. The WHO-estimated TB IR in the Philippines is high, and the NNS and NNT were high in our setting. NNT for immigrants from Pakistan and Thailand was similar, although the estimated TB IR is substantially lower in Thailand than in Pakistan. When eligibility for screening was based on TB IRs in countries of origin, NNT was fairly similar for the different thresholds and highest for those with IRs $>200 / 100$ 000, including Eritrea and Afghanistan. Estimates were lowest for immigrants from the Horn of Africa.

Only a small percentage (range $3 \%-21 \%$ ) of LTBI-positive immigrants were estimated to have received LTBI treatment (table 4). The resulting estimated number of incident TB cases prevented by LTBI treatment was therefore modest, with a limited overall public health impact of the immigrant LTBI screening programme in Norway in this period.

Almost half (range 30\%-58\%) of LTBI treatments were prescribed $>12$ months after arrival in Norway (table 4). The highest percentages were for immigrants from the Horn of Africa, where most incident TB occurs. A substantial proportion of additional incident TB cases could have been prevented if the same number of LTBI treatments had been prescribed sooner after arrival (table 4).

\section{DISCUSSION}

NNS and NNT to prevent one adverse outcome are measures used to communicate the effectiveness of healthcare interventions. ${ }^{18}$ In this study of the immigrant LTBI screening programme in Norway, we found overall very high NNS and NNT to prevent one incident TB case, and higher than in previous studies. ${ }^{419}$ Screening based on the TB NR in Norway rather than the TB IRs in source countries improved targeting of immigrants for LTBI management. However, NNS and NNT remained high for most countries by either approach, even when we applied the most optimistic estimates for test sensitivity, treatment effectiveness and treatment adherence.

\section{Strengths and limitations}

The strengths of this study include the availability of detailed country-specific administrative immigration and emigration data, which provide a strong estimate of the person-time observation for recent immigrants, the high sensitivity of the TB and LTBI surveillance system, and the performance of comprehensive sensitivity analyses for the different estimates. Given the availability of information on time in Norway prior to TB diagnosis or LTBI treatment from MSIS, we were able to demonstrate the effect of intervention timing. This approach has important clinical implications. Lastly, the overall consistency with the UK study ${ }^{4}$ makes comparison possible.

Study limitations include the currently weak monitoring and evaluation system of the Norwegian LTBI screening programme. Multiple service providers are involved in the screening process, with no harmonisation of data collection or follow-up documentation. Substantial delays in the provision of government-issued personal identification numbers to recent immigrants, specifically asylum seekers, have compromised follow-up and data linkage. For the same reason, we could not calculate NNT based on absolute risk reduction in LTBI-treated individuals. The lack of denominator data is a common challenge in most countries, which renders immigrant screening programmes poorly evaluated. We have used comprehensive administrative data and high-coverage surveillance data including information on LTBI treatment to overcome these limitations. 


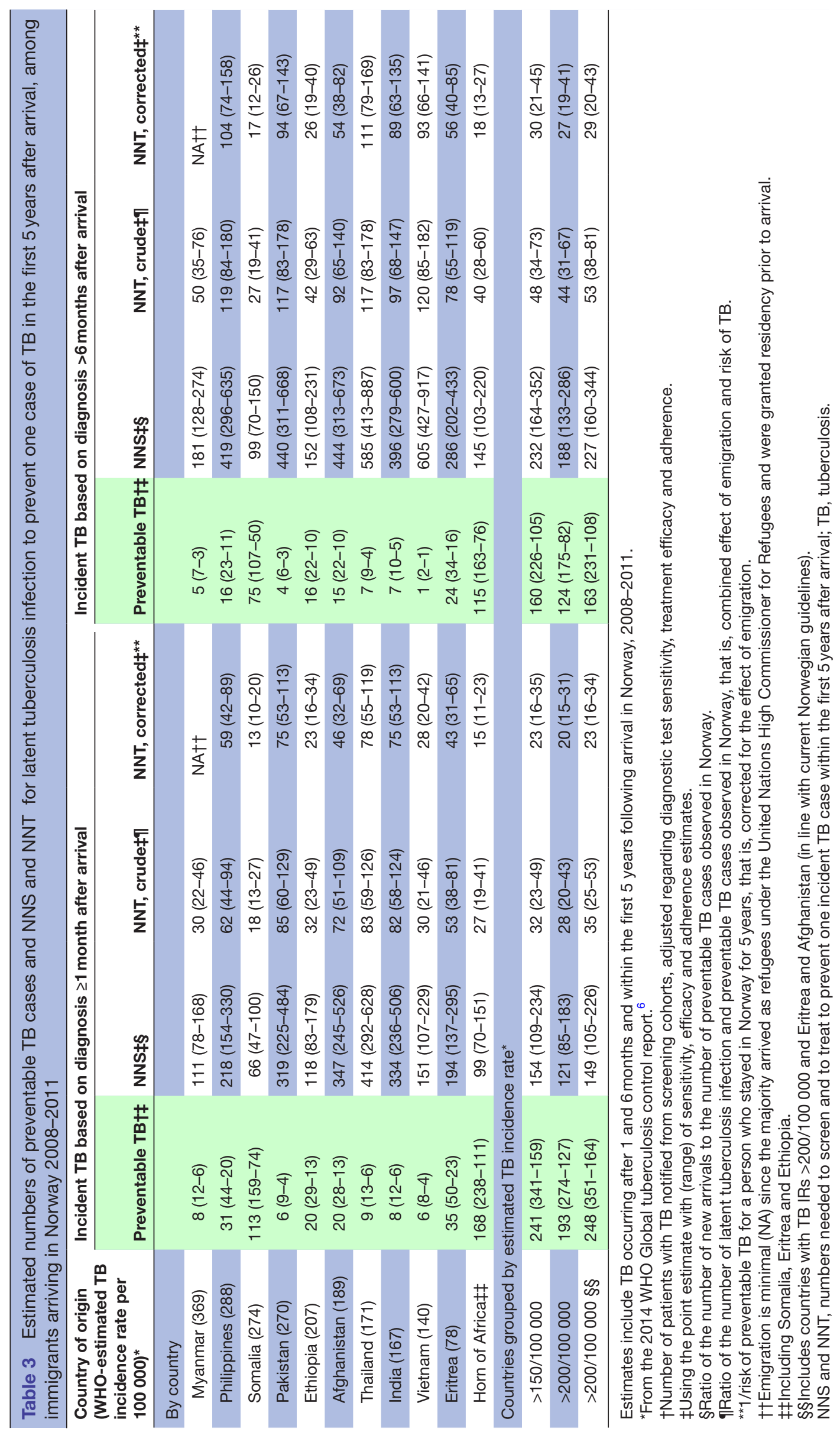




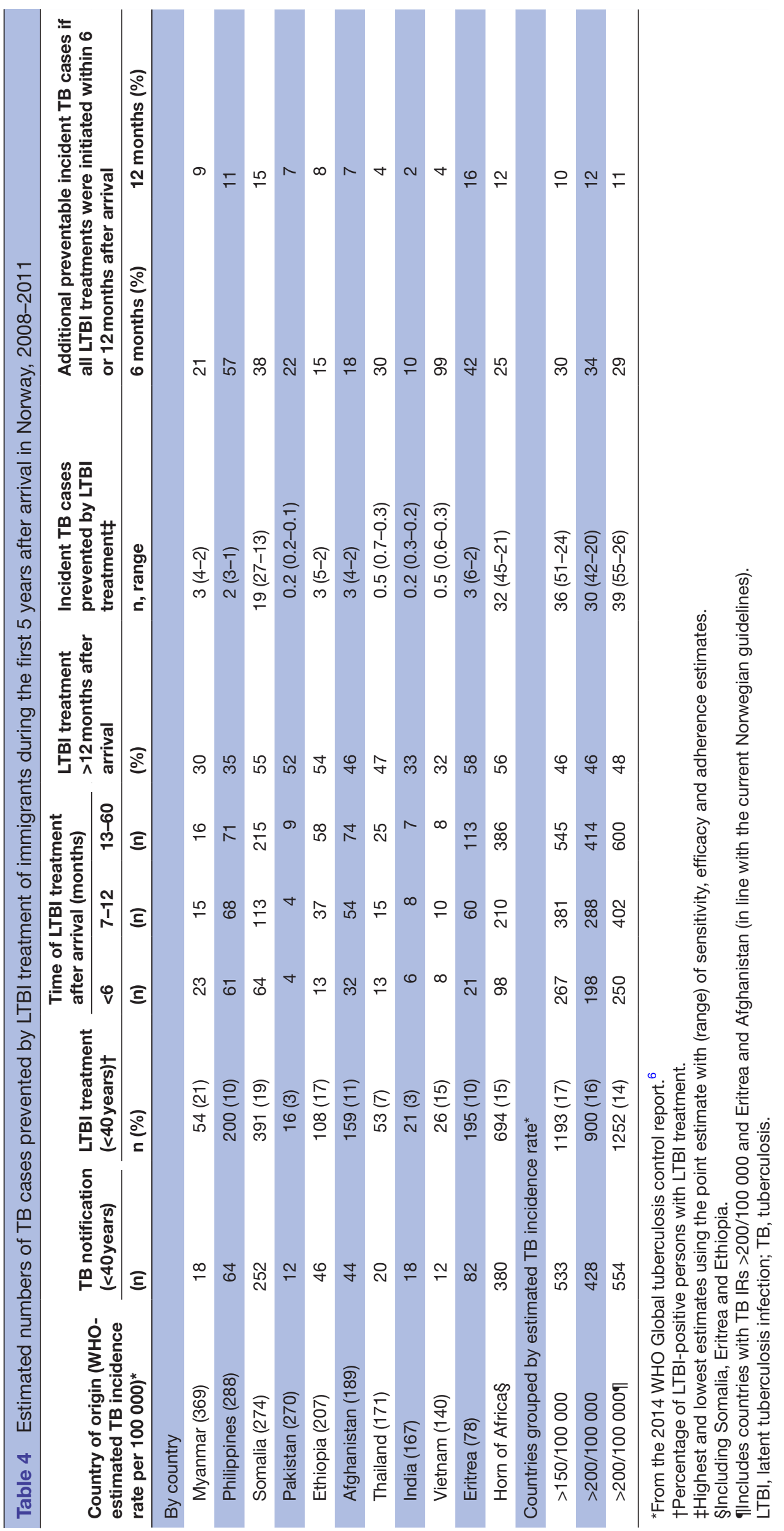


Screening coverage is high among asylum seekers and refugees, but less known for other immigrant groups (family reunification, students and immigrant workers). If screening participation was non-selective, it would not affect our estimates. However, if the prevalence of LTBI differed among those screened and not screened, our estimates may be biased.

The prevalence of LTBI in the arriving immigrant cohort was based on published literature, including Norwegian data on asylum seekers. ${ }^{8-10}$ Whether these correctly reflect the prevalence of LTBI in the arriving cohort is unknown, and this may potentially have biased our estimates in either direction. If the LTBI prevalence in the arriving immigrants was lower than estimated, the reported NNS and NNT would be too high, whereas with a higher prevalence than estimated our NNS and NNT would be too low.

Norwegian guidelines encourage treatment of individuals at greatest risk of progression to TB. If LTBI-positive individuals prescribed LTBI treatment were at greater risk than untreated LTBI-positive individuals, we may have underestimated the number of TB cases prevented by LTBI treatment during the study period. We may also have underestimated the overall benefit of the screening programme, as incident TB occurring $>5$ years after arrival was not included. However, whether incident TB occurring several years after arrival is related to initial infection or subsequent reinfection is difficult to evaluate in longterm follow-up studies. A Dutch study of molecular data in contacts showed that $83 \%$ of incident cases occurred within 5 years of the source case and $>95 \%$ occurred within 10 years, ${ }^{20}$ suggesting that the degree of potential underestimation was modest. Finally, the effects of screening for TB and LTBI are difficult to disentangle, as they contribute to each other.

\section{Comparison with other studies}

A UK study documented substantial variation in NNS and NNT among immigrants from the 10 most commonly reported source countries for TB in the UK. ${ }^{4}$ The figures contrasted with estimated TB IRs in the source countries. Similarly, we found great variation in NNS and NNT, which were not consistently related to estimated WHO TB IRs in source countries. Immigrants may originate from specific geographical areas with higher or lower rates than national averages, and their socioeconomic circumstances before and after arrival in host countries may differ. Surprisingly, the estimated NNT for source countries were overall considerably higher in Norway than in the UK. NNT for immigrants from Pakistan was $85(60-129)$ and 34 (17-70), from Somalia 18 (13-27) and $4(1-7)$, and from India $82(58-124)$ and 37 (20-61) in Norway and UK, respectively. ${ }^{4}$ In the current study, we differentiated between coprevalent and incident TB and accounted for emigration; both factors have profound impact on NNT and were not assessed in the UK study. ${ }^{4}$ Immigrants are screened soon after arrival in Norway, and many leave the country before the end of the 5-year observation period. In contrast, the UK study examined long-term immigrants. Differences in TB epidemiology may also contribute to the observed differences. The UK researchers reported higher TB rates, and higher transmission rates, than in most Western European countries, specifically in larger cities. ${ }^{21}$ The higher estimates for treatment adherence in this study compared with the UK study would narrow, rather than widen, the difference in NNT. A mathematical modelling study from Australia found that a combination of screening and subsequent treatment of all LTBI-positive immigrants would result in an overall reduction in the number of $\mathrm{TB}$ cases of about one-third to a half from 2013 to $2050 .{ }^{19}$ The NNS was 297 for all immigrants and 136 for immigrants originating from countries with an estimated TB IR >100/100 000, which is somewhat lower than in the current study. As in the UK study the model was based on permanent arrivals.

\section{Challenges of NNS/NNT estimation in immigrant screening}

The lifetime age-weighted risk of TB following infection in settings with low exogenous reinfection is estimated to be $12 \% .{ }^{22}$ The reported low pooled positive predictive value of the IGRA (2.7\%) corresponds to an NNT of 37 across different settings and populations. ${ }^{23}$ This corresponds to 111 months of treatment to prevent one TB case in need of 6 months of treatment. Thus, the risk reduction following LTBI treatment must be large to reduce the NNT. Although morbidity, mortality and transmission can be avoided if TB is prevented, the benefit of LTBI treatment for the individual should outweigh the risk of severe adverse effects. Although LTBI treatment is safe overall, it carries a risk of severe and potentially life-threatening toxic adverse effects. ${ }^{24}$

Register data did not allow us to clearly distinguish coprevalent TB from TB that developed later and was potentially preventable through LTBI management (incident TB). LTBI is considered to comprise a spectrum of infection states. ${ }^{25}$ A prolonged asymptomatic phase of early subclinical TB may precede clinical presentation with active disease. ${ }^{2627} \mathrm{~A}$ prearrival and postarrival evaluation of a cohort of US immigrants reported that $>80 \%$ of TB cases diagnosed within 1 year of receiving prearrival examination represented coprevalent $\mathrm{TB} .{ }^{27} \mathrm{~TB}$ diagnosed $<1$ month after arrival is clearly not preventable, whereas TB diagnosis within 1-6 months may or may not be preventable. Based on this uncertainty, we presented NNS and NNT separately for TB diagnosed $>1$ and $>6$ months after arrival.

Emigration was substantial in some groups. Immigrants to Norway from Myanmar were almost exclusively refugees under the United Nations High Commissioner for Refugees and were granted residency prior to arrival, whereas applications from adult asylum seekers from Afghanistan commonly were rejected. The observation years lost due to emigration were also substantial in other groups with high proportions of asylum seekers. Immigrants from the Philippines often arrive as au pairs and 
are granted only 2-year work permits. Emigration may also lead to NNT overestimation if immigrants who show LTBI positivity on screening on arrival in Norway develop TB after emigration.

\section{The effect of timeliness of screening and treatment}

In this study, less than one in five estimated LTBI-positive individuals (if all immigrants were screened) was treated. This gap in the intention to screen is intention to treat principle represents a challenge and has been reported in other Norwegian studies ${ }^{28-30}$; it has been due partly to Norwegian guidelines (in which the groups targeted for screening have been wider than those targeted for treatment), and measures have been taken to minimise it. ${ }^{7}$ It may, however, also signal that the number of LTBI-positive individuals is too high for the health services to treat, and/or that clinicians are reluctant to initiate LTBI treatment in individuals with unknown risk of progression to disease.

As a high proportion of incident TB cases occur early after arrival, an important component to improve the impact of the screening programme would be to ensure expedited follow-up and LTBI treatment initiation. Increased attention is given to the need for timely interventions as the incubation period for $\mathrm{TB} .{ }^{31}$ The reduced risk of progression to TB over time will increase NNT estimates with time, and delayed follow-up represents missed opportunities. The potential for additional prevented cases varied across countries of origin. The high potential for additional prevention among immigrants from Vietnam reflects the high proportions of those who are ill early after arrival and those for whom LTBI treatment is initiated late, whereas the opposite was observed for India.

\section{Comparing NNT with TB NR in Norway and WHO-estimated IRs in countries of origin}

We found a stronger numerical correlation between the NNT and TB NR in Norway than between the NNT and WHO-estimated IR in the country of origin for the top 10 source countries for TB in Norway. This is expected, as both the NRs and the NNT estimates are derived from the same Norwegian data (representing the same subset of the population who immigrated to Norway, which may not be a representative sample of the people in the country of origin), whereas the WHO-estimated IRs use country-specific data to make representative estimates for their national populations. When a large difference exists between the people in the country of origin and the subset of the population who immigrated to Norway, we would expect the TB NR in Norway to be more programmatically useful than the WHO-estimated IRs in countries of origin.

\section{Public health implications}

The overall high NNS and NNT in this study call into question whether routine LTBI screening of immigrants in a high-income low-incidence country is feasible, safe and effective, without the application of additional selection criteria. Although LTBI management based on TB notification in Norway rather than WHO-estimated IRs in countries of origin would have improved the targeting of immigrants, the NNS and NNT remained high.

The estimated number of incident TB cases prevented by LTBI treatment was modest, suggesting that substantial scale-up of the LTBI care cascade is necessary to strengthen the public health impact. Until new tests with higher predictive values for TB are available,$^{25}$ there are two complementary approaches to reduce the NNS and NNT. First, screening could be limited to immigrants with additional risk factors for disease, such as young age, recent known contact, abnormal X-ray findings and immunosuppressive conditions. This approach, however, will require additional resources to correctly identify risk groups on entry. Second, the LTBI care cascade could be improved so that further examinations and treatment are offered sooner following a positive LTBI screening test. The programme has the potential to prevent additional TB cases if more immigrants with LTBI are offered treatment, and this treatment starts sooner after arrival. TB disease develops usually 3-9months after exposure and rarely more than 2 years after exposure, ${ }^{31}$ which strengthens the recommendation for prompt follow-up of immigrant screening. A combination of these two approaches seems most plausible. Cost-effectiveness studies could help to identify the most beneficial approach in a Norwegian setting.

Monitoring of the effectiveness of screening should urgently be improved, by targeting immigrants with risk factors in addition to the TB IR in the source country and ensuring timely follow-up of screening. The data in Norway are better than in many other countries, but still with wide uncertainty. As immigration trends and composition and health services vary considerably among countries, better monitoring and evaluation of current screening programmes are needed so that countries can adjust their policies based on the yield of screening.

Even when applying the most optimistic estimates regarding diagnostic test sensitivity, treatment efficacy and adherence to treatment, a substantial proportion of incident TB cases will not be prevented through LTBI screening and management. Easy and equitable access to healthcare services for all should remain a cornerstone of TB control and prevention so that clinical cases are detected and treated early.

\section{Author affiliations}

${ }^{1}$ Department of Vaccine-Preventable Diseases, Norwegian Institute of Public Health, Oslo, Norway

${ }^{2}$ Department of Infectious Disease Epidemiology and Modelling, Norwegian Institute of Public Health, Oslo, Norway

${ }^{3}$ Statistics and Analysis Division, Norwegian Directorate of Immigration, 0slo, Norway

${ }^{4}$ Division of Infectious Diseases and Environmental Health, Norwegian Institute of Public Health, Oslo, Norway

${ }^{5}$ Institute of Health and Society, University of Oslo, Oslo, Norway

${ }^{6}$ Department of Tuberculosis, Blood-Borne and Sexually Transmitted Infections, Norwegian Institute of Public Health, Oslo, Norway 
Contributors BAW initiated the study, and BAW and EH wrote the protocol. BAW, RW and GMG were responsible for modelling and analyses. BAW, RW and EH drafted the manuscript. BAW, PK, PA, EH, RW and GMG provided input to discussions. All authors have read and approved the final version of the manuscript.

Funding BAW was funded by the Norwegian Health Association.

Competing interests None declared.

Patient consent for publication Not required.

Ethics approval Ethical approval of the study was obtained from the Regional Committee for Medical and Health Research Ethics, South-East Norway (2017/164).

Provenance and peer review Not commissioned; externally peer reviewed.

Data sharing statement Study data are available from the corresponding author on reasonable request.

Open access This is an open access article distributed in accordance with the Creative Commons Attribution Non Commercial (CC BY-NC 4.0) license, which permits others to distribute, remix, adapt, build upon this work non-commercially, and license their derivative works on different terms, provided the original work is properly cited, appropriate credit is given, any changes made indicated, and the use is non-commercial. See: http://creativecommons.org/licenses/by-nc/4.0/.

\section{REFERENCES}

1. WHO. Guidelines for the management of latent tuberculosis infection Geneva. 2014 http://www.who.int/tb/publications/ltbi_document_ page/en/.

2. WHO. Latent TB Infection: Updated and consolidated guidelines for programmatic management. 2018. WHO/CDS/TB/2018.4:74 http:// www.who.int/tb/publications/2018/latent-tuberculosis-infection/en/.

3. Kunst $\mathrm{H}$, Burman M, Arnesen TM, et al. Tuberculosis and latent tuberculous infection screening of migrants in Europe: comparative analysis of policies, surveillance systems and results. Int $J$ Tuberc Lung Dis 2017;21:840-51.

4. Kruijshaar ME, Abubakar I, Stagg HR, et al. Migration and tuberculosis in the UK: targeting screening for latent infection to those at greatest risk of disease. Thorax 2013;68:1172-4.

5. Arnesen TM, Heldal E, Mengshoel AT, et al. Tuberkulose i Norge $i$ 2016 med behandlingsresultater for 2015. Oslo: Folkehelseinstituttet, 2017.

6. WHO. Global tuberculosis report. Geneva, Switzerland, 2014

7. Folkehelseinstituttet. Guidelines for prevention and control of tuberculosis. Oslo: Norwegian Institute of Public Health, 2016.

8. Winje BA, Oftung F, Korsvold GE, et al. Screening for tuberculosis infection among newly arrived asylum seekers: comparison of QuantiFERONTB Gold with tuberculin skin test. BMC Infect Dis 2008;8:65.

9. Pareek M, Bond M, Shorey J, et al. Community-based evaluation of immigrant tuberculosis screening using interferon $\gamma$ release assays and tuberculin skin testing: observational study and economic analysis. Thorax 2013;68:230-9.

10. Pareek M, Watson JP, Ormerod LP, et al. Screening of immigrants in the UK for imported latent tuberculosis: a multicentre cohort study and cost-effectiveness analysis. Lancet Infect Dis 2011;11:435-44.
11. Pai M, Denkinger CM, Kik SV, et al. Gamma interferon release assays for detection of Mycobacterium tuberculosis infection. Clin Microbiol Rev 2014;27:3-20.

12. Diel R, Loddenkemper R, Nienhaus A. Evidence-based comparison of commercial interferon-gamma release assays for detecting active TB: a metaanalysis. Chest 2010;137:952-68.

13. Stagg HR, Zenner D, Harris RJ, et al. Treatment of latent tuberculosis infection: a network meta-analysis. Ann Intern Med 2014;161:419-28.

14. Erkens CG, Slump E, Verhagen M, et al. Monitoring latent tuberculosis infection diagnosis and management in the Netherlands. Eur Respir J 2016;47:1492-501.

15. Kan B, Kalin M, Bruchfeld J. Completing treatment for latent tuberculosis: patient background matters. Int J Tuberc Lung Dis 2013;17:597-602.

16. Olsen $\mathrm{Al}$, Andersen $\mathrm{HE}, \mathrm{ABmus} \mathrm{J}$, et al. Management of latent tuberculous infection in Norway in 2009: a descriptive crosssectional study. Public Health Action 2013;3:166-71.

17. Schein YL, Madebo T, Andersen HE, et al. Treatment completion for latent tuberculosis infection in Norway: a prospective cohort study. BMC Infect Dis 2018;18:587.

18. Laupacis A, Sackett DL, Roberts RS. An assessment of clinically useful measures of the consequences of treatment. N Engl J Med 1988;318:1728-33.

19. Denholm JT, McBryde ES. Can Australia eliminate TB? Modelling immigration strategies for reaching MDG targets in a lowtransmission setting. Aust N Z J Public Health 2014;38:78-82.

20. Borgdorff MW, Sebek M, Geskus RB, et al. The incubation period distribution of tuberculosis estimated with a molecular epidemiological approach. Int J Epidemiol 2011;40:964-70.

21. Kirby T. Tuberculosis rates unacceptably high in UK cities. Lancet Infect Dis 2013;13:836-7.

22. Vynnycky E, Fine PE. The natural history of tuberculosis: the implications of age-dependent risks of disease and the role of reinfection. Epidemiol Infect 1997;119:183-201.

23. Diel R, Loddenkemper R, Nienhaus A. Predictive value of interferon- $\gamma$ release assays and tuberculin skin testing for progression from latent TB infection to disease state: a meta-analysis. Chest 2012;142:63-75.

24. Kunst $\mathrm{H}$, Khan KS. Age-related risk of hepatotoxicity in the treatment of latent tuberculosis infection: a systematic review. Int $J$ Tuberc Lung Dis 2010;14:1374-81.

25. Esmail $\mathrm{H}$, Barry CE, Young DB, et al. The ongoing challenge of latent tuberculosis. Philos Trans R Soc Lond B Biol Sci 2014;369:20130437.

26. Dheda K, Barry CE. 3rd, Maartens G. Tuberculosis. Lancet 2016;387:1211-26.

27. Walter ND, Painter J, Parker M, et al. Persistent latent tuberculosis reactivation risk in United States immigrants. Am J Respir Crit Care Med 2014;189:88-95.

28. Harstad I, Jacobsen GW. Poor follow-up after screening for tuberculosis. TidsskrNor Laegeforen 2012;132:14-16.

29. Harstad I, Jacobsen GW, Heldal E, et al. The role of entry screening in case finding of tuberculosis among asylum seekers in Norway. BMC Public Health 2010;10:670.

30. Winje BA, White R, Syre $\mathrm{H}$, et al. Stratification by interferon- $\gamma$ release assay level predicts risk of incident TB. Thorax 2018:652-61.

31. Behr MA, Edelstein PH, Ramakrishnan L. Revisiting the timetable of tuberculosis. BMJ 2018;362:k2738. 